\title{
Possible Improvement of Liver Steatosis Associated with Elevated Creatine Phosphokinase Level Due to Subclinical Hypothyroidism by Thyroid Hormone Replacement Therapy
}

\author{
Satoshi Takakusagi ${ }^{1}$, Ken Sato*2, Yuhei Suzuki ${ }^{1}$, Takayoshi Kobayashi ${ }^{1}$, Satoru Kakizaki ${ }^{2}$ and Hitoshi \\ Takagi $^{1}$
}

${ }^{1}$ Department of Gastroenterology and Hepatology, Kusunoki Hospital, Japan

${ }^{2}$ Department of Gastroenterology and Hepatology, Gunma University Graduate School of Medicine, Japan

Received: April 15, 2018; Published: April 24, 2018

*Corresponding author: Ken Sato, Departmernt of Gastroenterology and Hapatology, Gunma University Graduate School of Medicine, 3-39-22 Showamachi, Maebashi, Gunma 371-8511, Japan

\section{Abstract}

A 71-year-old man suffering from non-alcoholic fatty liver disease, diabetes and dyslipidemia was hospitalized for elevated creatine phosphokinase levels. Discontinuation of antihyperlipidemic agents and rehydration could not normalize creatine phosphokinase levels. Findings of liver biopsy were compatible with non-alcoholic steatohepatitis. Based on the detailed evaluations, he was diagnosed as hypothyroidism. Thyroid hormone replacement therapy gradually improved his thyroid function and creatine phosphokinase levels. Notably, the repeated liver biopsy after the therapy showed histological improvement of steatosis. We should suspect "subclinical" hypothyroidism in case of unexplained elevation of creatine phosphokinase levels during the course or at the onset of non-alcoholic fatty liver/ non-alcoholic steatohepatitis.

Keywords: Hypothyroidism; Non-Alcoholic Steatohepatitis; Dyslipidemia; Creatinine Phosphokinase

Abbreviations: CK: Creatine Phosphokinase; TSH: Thyroid Stimulating Hormone; NASH: Non-Alcoholic Steatohepatitis; DL: Dyslipidemia; ALT: Alanine Aminotransferase; DM: Diabetes Mellitus; BMI: Body Mass Index; AST: Aspartate Aminotransferase; LDL: Low-Density Lipoprotein Cholesterol; HbA1c: Hemoglobin A1c; NAFL: Non-alcoholic Fatty Liver; VLDL: Very Low Density Lipoprotein; FGF-21: Fibroblast Growth Factor-21; ATP: Adenosine Triphosphate; HMG-CoA: Hydroxy Methyl Glutaryl-CoA

\section{Introduction}

There are few reports of elevation of creatine phosphokinase (CK) levels and rhabdomyolysis caused by hypothyroidism [13]. On the other hand, hypothyroidism and elevated thyroid stimulating hormone (TSH) levels are reportedly independent risk factors for non-alcoholic steatohepatitis (NASH) [4,5]. Herein, we report a valuable case of NASH in which the elevation of CK levels due to hypothyroidism developed after long-term use of statins for dyslipidemia (DL) and thyroid hormone replacement therapy was most likely to improve CK levels and liver steatosis.

\section{Case Report}

A 71 years-old man who had worked as a landscaper until 5 years ago was admitted to our hospital for the elevation of CK levels (2782 IU/mL, normal range: $0-180)$ and deterioration of both gastrocnemius muscle pains. His body height was $174 \mathrm{~cm}$ and body weight was $80.5 \mathrm{~kg}$ (BMI $26.6 \mathrm{~kg} / \mathrm{m}^{2}$ ), thus, he was thought to be obesity. His body temperature was normal $\left(96.4^{\circ} \mathrm{F}\right)$, and no abnormalities were also shown in other vital signs. He had no history of allergy, edema in lower limbs, goiter, hoarseness, and skin roughness. He had been treated hypercholesterolemia by statins for 11 years (pravastatin, atorvastatin, and rosuvastatin in this order) and add-on ezetimibe had been administered since 6 years before. Since 11 years before, his alanine aminotransferase (ALT) had been mildly elevated (approximately $50 \mathrm{IU} / \mathrm{L}$ ), and ultrasonography had kept showing fatty liver such as (Figure 1A). He had also been treated diabetes mellitus (DM) by glimepiride, alogliptin, and voglibose since 6 years before. Although self-reported dietary intake was not changed, his body weight was gradually increased (57 $\mathrm{kg}$ [body mass index (BMI) $18.8 \mathrm{~kg} / \mathrm{m}^{2}$ ] in 3 years ago, $62 \mathrm{~kg}$ [BMI $20.5 \mathrm{~kg} / \mathrm{m}^{2}$ ] in a year ago, and $64 \mathrm{~kg}$ [BMI $\left.21.1 \mathrm{~kg} / \mathrm{m}^{2}\right]$ in 5 months ago). Therefore, nutrition intervention was performed, but it was not effective. In addition, he had sometimes felt mild both gastroc- 
nemius muscle pains without specific factors such as exercise since 10 years before, and his CK levels had been slightly elevated (approximately $400 \mathrm{IU} / \mathrm{L})$.

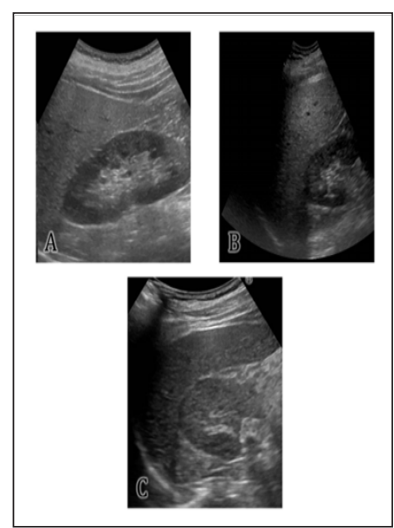

Figure 1: The change of the findings of abdominal ultrasonography during the course.

a. Hepato-renal echo contrast that is typically observed in fatty liver 4 months before admission.

b. The similar finding was observed at the time of admission.

c. Hepato-renal echo contrast was almost disappeared on 108 days after discharge from the hospital.

At the time of admission, his laboratory data showed a significant elevation of CK levels, but CK-MB and myoglobin levels were within normal range. In addition, aspartate aminotransferase (AST), ALT, lactate dehydrogenase, $\gamma$-glutamyltransferase, total cholesterol, low-density lipoprotein cholesterol (LDL), triglyceride, and hemoglobin A1c (HbA1c) levels were also elevated (Table 1). Physical examination showed no abnormality including goiter. Abdominal ultrasonography showed fatty liver same as before (Figure 1B). Although aggressive excise was not likely to be performed and he seemed not to spend outside so long hours just before hospitalization, myopathy due to dehydration based on high outdoor air temperature (the lowest and highest temperature in Gunma prefecture from one day before his hospitalization to the hospitalization day were about $77^{\circ} \mathrm{F}$ and $95^{\circ} \mathrm{F}$, respectively.) was suspected.

Table 1: Laboratory Data on admission.

\begin{tabular}{|c|c|c|c|}
\hline \multicolumn{2}{|c|}{ Hematology } & \multicolumn{2}{c|}{ Biochemistry } \\
\hline $\mathrm{Hb}$ & $13.3 \mathrm{~g} / \mathrm{dL}$ & $\mathrm{TP}$ & $7.6 \mathrm{~g} / \mathrm{dL}$ \\
\hline $\mathrm{RBC}$ & $419 \times 10^{4} / \mu \mathrm{L}$ & Alb & $5 \mathrm{~g} / \mathrm{dL}$ \\
\hline $\mathrm{Ht}$ & $39.7 \%$ & $\mathrm{~T}-\mathrm{Bil}$ & $0.7 \mathrm{mg} / \mathrm{dL}$ \\
\hline $\mathrm{WBC}$ & $7920 / \mu \mathrm{L}$ & $\mathrm{D}-\mathrm{Bil}$ & $0.1 \mathrm{mg} / \mathrm{dL}$ \\
\hline Neu & $37.4 \%$ & AST & $90 \mathrm{IU} / \mathrm{L}$ \\
\hline Lym & $50.1 \%$ & ALT & $66 \mathrm{IU} / \mathrm{L}$ \\
\hline Eos & $6.4 \%$ & LDH & $314 \mathrm{IU} / \mathrm{L}$ \\
\hline Mono & $4.3 \%$ & ALP & $143 \mathrm{IU} / \mathrm{L}$ \\
\hline Baso & $0.9 \%$ & $\gamma$-GTP & $69 \mathrm{IU} / \mathrm{L}$ \\
\hline PLT & $21.3 \times 10^{4} / \mu \mathrm{L}$ & $\mathrm{CK}$ & $2782 \mathrm{IU} / \mathrm{L}$ \\
\hline
\end{tabular}

\begin{tabular}{|c|c|c|c|}
\hline \multicolumn{2}{|c|}{ Coagulation } & CK-MB & $19 \mathrm{IU} / \mathrm{L}$ \\
\hline PT & $79.2 \%$ & myoglobin & $135 \mathrm{ng} / \mathrm{mL}$ \\
\hline \multicolumn{2}{|c|}{ Urinalysis } & BUN & $16.3 \mathrm{mg} / \mathrm{dL}$ \\
\hline specific gravity & 1.018 & $\mathrm{Cr}$ & $1.2 \mathrm{mg} / \mathrm{dL}$ \\
\hline pH & 5.5 & $\mathrm{Na}$ & $139 \mathrm{mEq} / \mathrm{L}$ \\
\hline urinary protein & $(-)$ & $\mathrm{K}$ & $4.4 \mathrm{mEq} / \mathrm{L}$ \\
\hline urinal sugar & $(-)$ & $\mathrm{C}$ & $105 \mathrm{mEq} / \mathrm{L}$ \\
\hline occult blood & $(-)$ & CRP & $0.1 \mathrm{mg} / \mathrm{dL}$ \\
\hline ketone body & $(1+)$ & Glu & $228 \mathrm{mg} / \mathrm{dL}$ \\
\hline urobilinogen & normal & T-C & $239 \mathrm{mg} / \mathrm{dL}$ \\
\hline bilirubin & $(-)$ & LDL-C & $172 \mathrm{mg} / \mathrm{dL}$ \\
\hline & & HDL-C & $37 \mathrm{mg} / \mathrm{dL}$ \\
\hline & & Triglyceride & $236 \mathrm{mg} / \mathrm{dL}$ \\
\hline & & HbA1c & $7.3 \%$ \\
\hline
\end{tabular}

Note: $\gamma$-GTP, y-glutamyltransferase; Alb, albumin; ALP, alkaline phosphatase; ALT, alanine aminotransferase; AST, aspartate aminotransferase; Baso, basophils; BUN, blood urea nitrogen; $\mathrm{CK}$, creatine phosphokinase; $\mathrm{Cr}$, creatinine; CRP, C-reactive protein; D-Bil, direct bilirubin; Eos, eosinophils; Glu, glucose; $\mathrm{Hb}$, hemoglobin; HbA1c, hemoglobin A1c; HDL-C, high density lipoprotein cholesterol; $\mathrm{Ht}$, hematocrit; $\mathrm{LDH}$, lactate dehydrogenase; LDL-C, low density lipoprotein cholesterol; Lym, lymphocytes; Mono, monocytes ; Neu, neutrophils; Plt, platelets; PT, prothrombin; RBC, red blood cell count; T-Bil, total bilirubin; T-C, total cholesterol; $\mathrm{TP}$, total protein; WBC, white blood cell count.

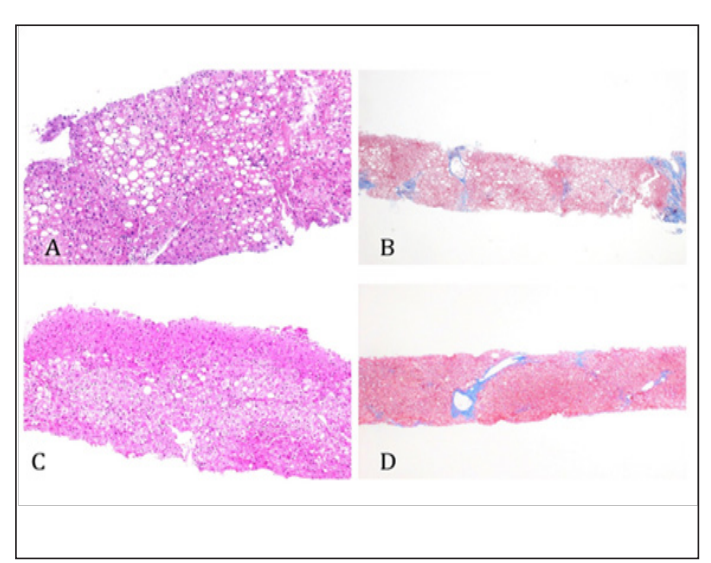

Figure 2: Pathological findings of the liver specimens on day 12 of admission (A and B) and on 108 days after discharge from the hospital (C and $D)$. Liver biopsy specimen on day 12 of admission (A and B) showed grade 1 and stage 2 non-alcoholic steatohepatitis (NASH) by Brunt' s classification. Approximately $30 \%$ steatosis was observed (B). Liver steatosis was clearly reduced to $5-10 \%$ on 108 days after discharge from the hospital (D). Hematoxylin-eosin staining $(\times 100)(\mathrm{A}$ and $\mathrm{C})$. Masson trichrome staining $(\times 4)(\mathrm{B}$ and $\mathrm{D})$.

Therefore, we started to perform rehydration therapy (1000 to $1500 \mathrm{ml} /$ day). In addition, rosuvastatin and ezetimibe were 
discontinued because those might have caused myopathy. As a result, CK levels tended to be decreased, but it had been remained around $1000 \mathrm{IU} / \mathrm{L}$ from days 5 to 10 of admission. AST and ALT levels were also increased. On day 12 of admission, we suspected the elevation of CK levels due to hypothyroidism. Then, we found that thyroid stimulating hormone (TSH) levels were elevated $(234 \mu \mathrm{U} /$ $\mathrm{mL}$ ) and free T3 and free T4 levels were below the detection limit. Besides, anti-thyroglobulin antibody and anti-thyroid peroxydase antibody were also positive. Thyroid ultrasonography showed the hypoechogenicity and heterogeneity of thyroid gland. Based on these results, he was diagnosed as chronic thyroiditis (Hashimoto's disease). Liver biopsy performed for the evaluation about the cause of increased transaminase levels showed 30\% steatosis, balloon-like change of hepatocytes, pericellular fibrosis, and portal fibrosis. Thus, he was diagnosed as NASH with grade 1 and stage 2 by Brunt's classification (Figures 2A \& 2B) [6]. As thyroid hormone replacement therapy, levothyroxine was started at $25 \mu \mathrm{g} /$ day and was gradually increased. As a result, thyroid function, CK levels, ALT levels, and muscle pain were improved, and he was discharged on day 40 of admission. On day 73 after discharge from the hospital, ALT levels were elevated to $115 \mathrm{IU} / \mathrm{L}$ and TSH levels remained at $71 \mu \mathrm{IU} / \mathrm{mL}$ although $\mathrm{CK}$ levels were improved. Therefore, levothyroxine was increased to $75 \mu \mathrm{g} /$ day. Later, ALT and TSH levels were improved. On day 109 after discharge from the hospital, his body weight and BMI were improved to $71.9 \mathrm{~kg}$ and $23.7 \mathrm{~kg} / \mathrm{m}^{2}$, respectively. LDL cholesterol and triglyceride levels were fluctuated after the discontinuation of rosuvastatin and ezetimibe but gradually decreased with increase of levothyroxine. Triglyceride levels reached to the normal range while LDL cholesterol levels almost reached to same levels as before the admission without antihyperlipidemic agents. HbA1c levels were gradually decreased after admission, and finally reached to the lower levels compared to those before admission without increase of oral hypoglycemic agents. Second liver biopsy showed the improvement of steatosis (Figures 2C \& 2D), and abdominal ultrasonography also showed the improvement of hepato-renal echo contrast (Figure 1C). The clinical course is shown in (Figure 3).

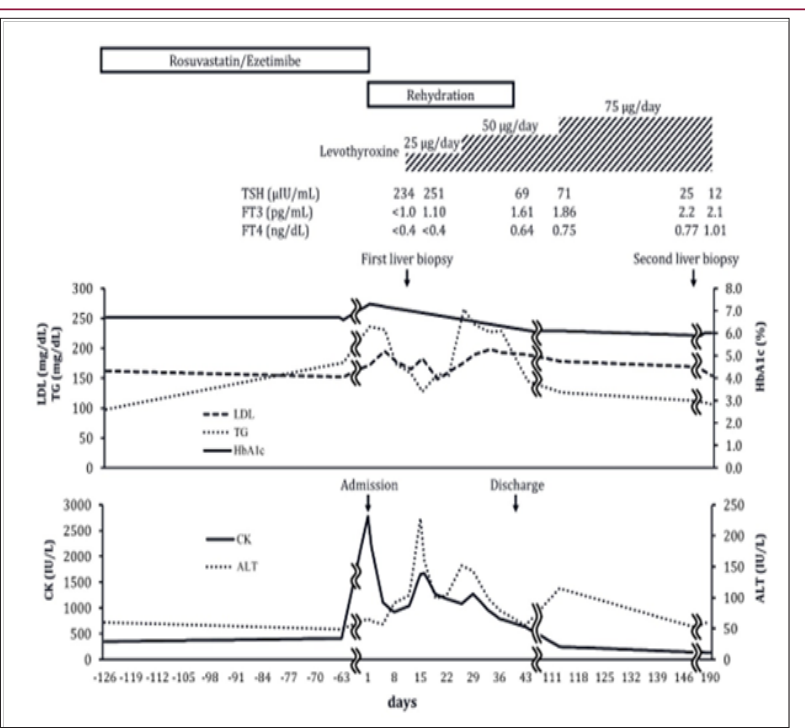

Figure 3: The clinical course of the patient. Because of the elevation of CK levels $(2782 \mathrm{IU} / \mathrm{mL})$, we discontinued the administration of antihyperlipidemic agents that were rosuvastatin and ezetimibe. Besides, we started to perform rehydration therapy. Resultantly, CK levels tended to be decreased but was not normalized. We performed first liver biopsy to evaluate the cause of prolonged abnormality of transaminase levels. We suspected that elevated CK levels were due to hypothyroidism and found that elevated thyroid stimulating hormone (TSH), low Free T3, and T4 levels on day 12 of admission. Then, we started to administer $25 \mu \mathrm{g} /$ day levothyroxine. As serum CK and ALT levels were fluctuated, we increased the dose of levothyroxine up to $50 \mu \mathrm{g} /$ day and resultantly, both levels were gradually decreased. However, serum ALT levels increased again. Then we finally increased the dose of levothyroxine up to $75 \mu \mathrm{g} /$ day and serum ALT levels gradually decreased. HbA1c levels were gradually decreased after admission, and finally reached to the lower level compared to those before admission. Triglyceride and LDL levels were increased after discontinuation of rosuvastatin and ezetimibe and fluctuated for a while. Those levels were gradually decreased during the administration of $50 \mu \mathrm{g} /$ day levothyroxine. Triglyceride levels reached to the normal range while LDL cholesterol levels almost reached to those prior to admission without antihyperlipidemic agents. Regarding thyroid function, TSH levels were gradually decreased and, Free T3 and T4 levels were gradually increased after the administration of levothyroxine. Later, Free T3 and T4 levels were reached to the normal levels.

\section{Discussion}

The major findings from our case are as follows.

a. Our case was likely to be secondary NASH due to hypothyroidism. b. Thyroid hormone replacement therapy was likely to ameliorate not only CK levels but also lifestyle diseases.

c. Histological improvement of hepatic steatosis could be confirmed by the comparison of biopsies before and after thyroid hormone replacement therapy. 
Non-alcoholic fatty liver (NAFL) has been reported to be closely related to obesity, impaired glucose tolerance, DL, and hypertension. It has been positioned as liver lesion of metabolic syndrome based on insulin resistance [7]. In our case as well, the patient diagnosed as DL and NAFL 11 years before, and both of those had been thought to be developed with metabolic syndrome until his admission. However, there are secondary NAFL/NASH and the primary diseases of secondary NAFL/NASH including endocrine diseases such as hypothyroidism, pituitary dysfunction, gonadal dysfunction, polycystic ovary syndrome, obstructive sleep apnea, kwashiorkor, fatty acid $\beta$ oxidative disorder, very low-density lipoprotein (VLDL) synthetic secretion abnormality, metabolic disorder after pancreaticoduodenectomy, and drug-induced metabolic disorder [4]. As hypothyroidism often causes increase of CK levels, we suspected hypothyroidism as primary diseases of secondary NAFL. In fact, the liver specimens were compatible with NASH, and as expected, he was also diagnosed as hypothyroidism based on laboratory and imaging data although no characteristic symptoms of hypothyroidism such as edema in the lower limb, hoarseness, and skin roughness were observed. In addition, thyroid hormone replacement therapy improved hepatic fat accumulation, CK levels, DL, body weight and BMI. The self-reported total calories consume was not changed and the caloric restriction with nutritional guidance had not been performed after thyroid hormone replacement therapy. Thus, the improvement of clinical condition was not likely to be due to lifestyle change.

Besides, other primary diseases that could cause secondary NASH was unlikely based on his past medical history, life history, medication history, nutritional status, adrenocorticotrophic hormone and cortisol levels, respiratory state during his hospitalization and so on. Unfortunately, as we had not measured thyroid function before the admission, the onset of hypothyroidism was unknown. However, he might have suffered from "subclinical" hypothyroidism since approximately one decade before because mild elevation of CK levels and muscle pain had been developed since one decade before. Besides, the disease duration of NAFL was similar to that of "subclinical" hypothyroidism. Thus, our case was likely to be secondary NASH based on hypothyroidism. Pagadala et al.[8] compared 246 NAFL patients with 430 control groups matched for age, sex, race, BMI, and reported that the prevalence of hypothyroidism was significantly higher among NAFL patients than controls. Furthermore, they also reported that the prevalence of hypothyroidism was significantly higher among NASH patients than non-NASH patients in NAFL patients.

Although the clear mechanism to develop NAFL/NASH from hypothyroidism is still unknown, the enhancement of general mechanism in metabolic syndrome is assumed. That is, the presumptive mechanisms include relative overnutrition due to hypothyroidism causes obesity, insulin resistance, lipid metabolism disorder, adipocytokine abnormality, oxidative stress, lipid peroxidation, and inflammatory cytokine. For example, regarding adipocytokines, especially, leptin plays an important role in appetite regulation, and induces collagen production and insulin resistance in liver [9-11]. Leptin was reported to be elevated due to hypothyroidism, which is considered to be a cause leading to NAFL/ NASH $[12,13]$. In addition, thyroid hormone has been thought to suppress the increase of weight, LDL levels and triglyceride through $\beta$ receptor expressed in liver $[14,15]$. Liver steatosis was reportedly improved by liver-targeted thyroid hormone receptor agonists [16]. Furthermore, the involvement of fibroblast growth factor-21 (FGF-21) in NAFL/NASH has been considered. FGF-21 is one of FGF family and has hormone-like effect. The improvement of insulin resistance by FGF-21 [17,18], and the induction of liver-specific FGF-21 by administration of thyroid hormone was reported [19]. Therefore, hypothyroidism was likely to cause the reduced effect of FGF-21, and lead to the development of NAFL/NASH.

On the other hand, the prevalence of muscle-related complications in adults with hypothyroidism is high, and CK levels were reportedly elevated in 57 to $90 \%$ cases [3]. It has been assumed that the conversion from type II muscle fiber (fast muscle) to type I muscle fiber (slow muscle), accumulation of glycosaminoglycan, reduction of contraction activity of actin-myosin, reduction of ATP (adenosine triphosphate) metabolism, myosin ATPase activity, hypoxia, energy storage reduction and so on seemed to be mechanisms by which myopathy is caused due to hypothyroidism [1]. Further, in cases of hypothyroidism, the prevalence of elevated CK levels was increased to 5 times by receiving of statin [20], although majority of rhabdomyolysis were caused by exercise and trauma [1,2]. In our case, based on undiagnosed hypothyroidism and statin use, it was thought that dehydration due to summer high temperature caused sudden elevation of CK levels. This consideration was supported with the clinical course in which the mild improvement of CK levels with hospitalization, rehydration and statin discontinuance, and rapid improvement of $\mathrm{CK}$ levels with levothyroxine replacement treatment were observed.

Thyroid hormone increases LDL receptors on the liver surface and cholesterol uptake from the blood. In addition, thyroid hormone induced hydroxymethylglutaryl-CoA (HMG-CoA) synthase and HMG-CoA reductase, and resultantly cholesterol synthesis from acetyl CoA is accelerated and cholesterol in hepatocyte increases. Patients with hypothyroidism, in which these mechanisms are impaired, frequently develops hypercholesterolemia. HMG-CoA reductase involved in endogenous cholesterol synthesis, however, is rather decreasing in those with hypothyroidism compared to those with normal thyroid function. Therefore, statins are not effective, and rather might increase the risk of adverse effects such as rhabdomyolysis [21], and thus, it should be avoided to administer until the thyroid function is normalized. In our case as well, diagnosis and treatment of hypothyroidism should have been performed earlier than receiving statin.

The limitation of our study is the lack of measurement of isozyme analysis of CK and insulin levels. Macro-creatine kinase should be differentiated in case of elevated CK levels. Cases of hypothyroidism associated with immunoglobulin-linked macro-creatine kinase are extremely rare but have been reported and macro-creatine kinase is reportedly disappeared during the course of thyroid hormone 
replacement therapy [22]. Besides, we could not evaluate insulin resistance due to the lack of measurement of insulin.

In conclusion, we experienced a NAFL case which developed the elevation of CK levels and was finally diagnosed as hypothyroidism and NASH. Thyroid hormone replacement therapy was most likely to cause the histological improvement of liver steatosis based on the clinical course. The further delay of the diagnosis and treatment for hypothyroidism would worse the clinical condition in our case. Hypothyroidism complicated by NAFL/NASH is assumed to be relative rare. However, we should suspect "subclinical" hypothyroidism in case of unexplained elevation of CK levels during the course or at the onset of NAFL/NASH.

\section{References}

1. Kisakol G, Tunc R, Kaya A (2003) Rhabdomyolysis in a patient with Hypothyroidism. Endocr J 50(2): 221-223.

2. Barahona MJ, Mauri A, Sucunza N, Paredes R, Wagner AM, et al. (2002) Hypothyroidism as a cause of Rhabdomyolysis. Endocr J 49(6): 621-623.

3. Kuo HT, Jeng CY (2010) Overt hypothyroidism with rhabdomyolysis and myopathy: a case report. Chin Med J (Engl) 123(5): 633-637.

4. Chalasani N, Younossi Z, Lavine JE, Diehl AM, Brunt EM, et al. (2012) The diagnosis and management of non-alcoholic fatty liver disease: practice guideline by the American gastroenterological association, American association for the study of liver diseases, and American college of gastroenterology. Gastroenterology 142(7): 1592-1609.

5. Carulli L, Ballestri S, Lonardo A, Lami F, Violi E, et al. (2013) Is nonalcoholic steatohepatitis associated with a high-though-normal thyroid stimulating hormone level and lower cholesterol levels? Intern Emerg Med 8(4): 297-305.

6. Brunt EM, Janney CG, Di Bisceglie AM, Neuschwander-Tetri BA, Bacon BR (1999) Nonalcoholic steatohepatitis: a proposal for grading and staging the histological lesions. Am J Gastroenterol 94(9): 2467-2474.

7. Ikeshima K (2016) Complicated etiology and pathology of NAFLD. Nihon Naika Gakkai Zasshi (Journal of Japanese Society of Internal Medicine) 105(1): 15-24 (in Japanese).

8. Pagadala MR, Zein CO, Dasarathy, Yerian LM, Lopez R, et al. (2012) Prevalence of hypothyroidism in nonalcoholic fatty liver disease. Dig Dis Sci 57(2): 528-534.

9. Oswai A, Yeo G (2010) Leptin and the control of body weight: a review of its diverse central targets, signaling mechanisms, and role in the pathogenesis of obesity. Obesity (Silver Spring) 18(2): 221-229.
10. Saxena NK, Ikeda K, Rockey DC, Friedman SL, Anania FA (2002) Leptin in hepatic fibrosis: evidence for increased collagen production in stellate cells and lean littermates of ob/ob mice. Hepatology 35(4): 762-771.

11. Benomar Y, Wetzler S, Larue Achagiotis C, Djiane J, Tomé D, et al. (2005) In vivo leptin infusion impairs insulin and leptin signaling in liver and hypothalamus. Mol Cell Endocrinol 242(1-2): 59-66.

12. Leonhardt U, Ritzel U, Schäfer G, Becker W, Ramadori G (1998) Serum leptin levels in hypo-and hyperthyroidism. J Endocrinol 157(1): 75-79.

13. Kautzky Wliier A, Ludwig C, Nowotny P, Roden A, Huemer C, et al. (1999) Elevation of plasma leptin concentrations in obese hyperinsulinaemic hyperthyroidism before and after treatment. Eur J Clin Invest 29(5): 395-403.

14. Grover GJ, Mellström K, Ye L, Malm J, Li YL, et al. (2003) Selective thyroid hormone receptor- $\beta$ activation: A strategy for reduction of weight, cholesterol, and lipoprotein (a) with reduced cardiovascular liability. Proc Natl Acad Sci U S A 100(17): 10067-10072.

15. Erion MD, Cable EE, Ito BR, Jiang H, Fujitaki JM, et al. (2007) Targeting thyroid hormone receptor- $\beta$ agonists to the liver reduces cholesterol and triglycerides and improves the therapeutic index. Proc Natl Acad Sci U S A 104(39): 15490-15495.

16. Cable EE, Finn PD, Stebbins JW, Hou J, Ito BR, et al. (2009) Reduction of hepatic steatosis in rats and mice after treatment with a liver-targeted thyroid hormone receptor agonist. Hepatology 49(2): 407-417.

17. Kharitonenkov A, Wroblewski VJ, Koester A, Chen YF, Clutinger CK, et al. (2007) The metabolic state of diabetic monkeys is regulated by fibroblast growth factor-21. Endocrinology 148(2): 774-781.

18. Xu J, Lloyd DJ, Hale C, Stanislaus S, Chen M, et al. (2009) Fibroblast growth factor 21 reverses hepatic steatosis, increases energy expenditure, and improves insulin sensitivity in diet-obese mice. Diabetes 58(1): 250259.

19. Adams AC, Astapova I, Fisher FM, Badman MK, Kurgansky KE, et al. (2010) Thyroid hormone regulates hepatic expression of fibroblast growth factor 21 in a PPAR $\alpha$-dependent manner. J Biol Chem 285(19): 14078-14082.

20. Tokinaga K, Oeda T, Suzuki Y, Matsushima Y (2006) HMG-CoA reductase inhibitor (statins) might cause high elevation of creatine phosphokinase (CK) in patients with unnoticed hypothyroidism. Endocr J 53(3): 401405 .

21. Kubota K (2008) Hypercholesterolemia and hypothyroidism. Sogo Rinsho (Clinic All-Round) 57(3): 448-452 (in Japanese).

22. Kageyama Y and Saitoh T (1996) A case of hypothyroidism associated with immunoglobulin-linked macro creatinine kinase. IRYO 50(12): 844-848 (in Japanese with English abstract).
This work is licensed under Creative Commons Attribution 4.0 License

Submission Link: https://biomedres.us/submit-manuscript.php

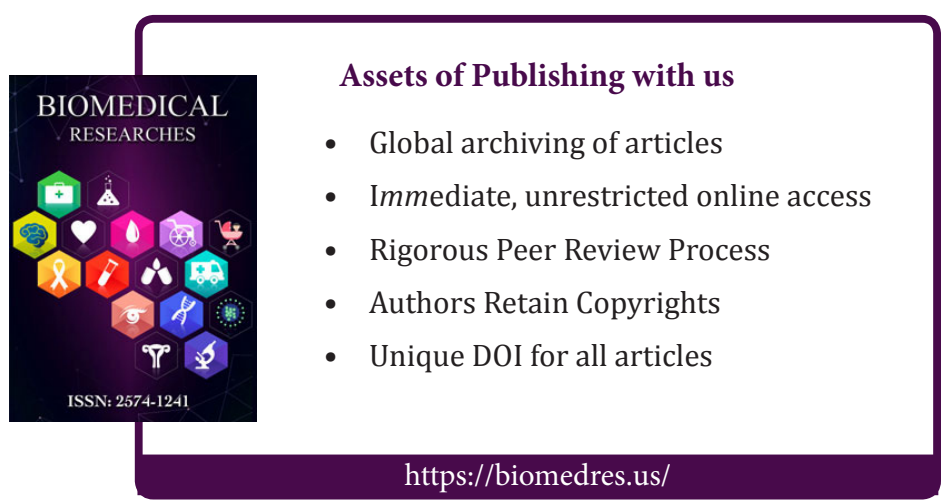

